\title{
Novel concept for the aggregation structure of fatty acid monolayers on the water surface and direct observation of molecular arrangements in their monolayers
}

\author{
TISATO KAJIYAMA and TAISHI KURI \\ Department of Chemical Science and Technology, Faculty of Engineering, Kyushu Univer- \\ sity, 6-10-1 Hakozaki, Higashi-ku, Fukuoka 812, Japan
}

\begin{abstract}
The aggregation structure of fatty acid monolayers on water subphases of different $\mathrm{pH}$ 's was investigated by means of transmission electron microscopy. Fatty acid monolayers exhibited the phase transition from an amorphous state to a crystalline one by surface compression in the case of a highly dissociated state of hydrophilic groups, whereas they did not show the phase transition in the case of a slightly dissociated state. The aggregation structure of monolayers on the water surface was systematically classified into "the crystalline monolayer", "the amorphous monolayer" and "the compressing crystallized monolayer" with respect to thermal and chemical (intermolecular repulsive) factors.

Molecular-resolution images of fatty acid molecules in the monolayers on mica substrate were successfully observed with an atomic force microscope (AFM) for the first time. The AFM image of a lignoceric acid monolayer prepared at a surface pressure of $5 \mathrm{mN} \mathrm{m}^{-1}$ showed a two-dimensional periodic structure with locally disordered molecular arrangements. Also, the nondestructive AFM image observation was successful for a stearic acid monolayer which was prepared by a multistep creep method, indicating that a high mechanical stability of the monolayer is inevitably required for the nondestructive AFM observation.
\end{abstract}

Keywords. Aggregation structure of monolayer; thermal and chemical factors; molecularresolution AFM image; nondestructive AFM observation.

\section{Introduction}

Great attention has been paid to Langmuir-Blodgett (LB) films because of their potential applications as electronic or electro-optical devices and as model biological membranes. The images of monolayers on water surface have been proposed on the basis of recent morphological and structural studies (Kjaer et al 1989; Kajiyama et al 1992). These images did not always agree with the general concept (see, for example, Gaines 1966) which was accepted only from surface pressure-area $(\pi-A)$ isotherms and also did not come to universal understanding. Here we present a novel and systematic classification for the aggregation structure of monolayers on water surface.

Fatty acid monolayers on pure water surface have been classified into a crystalline monolayer and an amorphous one at the subphase temperature $\left(T_{\mathrm{sp}}\right)$ below and above the melting temperature $\left(T_{\mathrm{m}}\right)$ of the monolayer, respectively. These aggregation states are independent of the magnitude of the surface pressure. The $\pi-A$ isotherm for the fatty acid monolayer represents the aggregating process of isolated domains grown right after spreading a fatty acid solution on water surface. On the other hand, phosphatidylcholine, phosphatidic acid and anionic amphiphile formed a compressing crystallized monolayer which was crystallized by compression of the monolayer on water surface at $T_{\mathrm{sp}}$ below $T_{\mathrm{m}}$ (Kajiyama et al 1993). For these cases, a fairly high surface pressure was required to crystallize the amphiphilic molecules with ionic hydrophilic groups owing to strong repulsion among ionic charges of hydrophilic groups. In order to understand systematically the aggregation structure of monolayers, 
it is necessary to investigate the effect of ionic repulsion among hydrophilic groups with respect to the aggregation structure of the monolayer on water surface.

An atomic force microscope (AFM) allows one to characterize the surface structure of organic materials on a molecular level. The molecular arrangements (Schwartz et al 1993) and structural defects (Peltonen et al 1992) of multilayered LB films were observed by using an AFM. On the other hand, the molecular-resolution AFM image of monolayers was successfully taken by using only limited samples such as a polymerized amphiphilic monolayer, dialkyl amphiphilic monolayer and fatty acid salt monolayer which were formed owing to a relatively stronger aggregation force. There has been no observation of the molecular-resolution image of the fatty acid monolayer with a weaker aggregation force.

In this study, we investigate the effect of ionic repulsion among hydrophilic groups with respect to the aggregation structure of the monolayer on water surface and also present the molecular-resolution AFM images of lignoceric and stearic acid monolayers on mica for the first time.

\section{Experimental}

\subsection{Analyses of the aggregation structure of monolayer on water surface}

Arachidic acid monolayers were prepared from a benzene solution on water subphases of pH 5.8 (pure water) and 12.6 (adjusted by addition of $\mathrm{NaOH}$ ) at $T_{\mathrm{sp}}$ of $303 \mathrm{~K}$ below $T_{m}(=328 \mathrm{~K})$. The ionic dissociation state of the hydrophilic group was estimated on the basis of the stretching vibrations of carbonyl and carboxylate groups by Fourier transform infrared attenuated total reflection (FT-IR ATR) measurements (Kajiyama et al 1993). Seventy arachidic acid monolayers were transferred on a germanium ATR prism, resulting in the formation of the multilayered film. Transfer on the prism was carried out at surface pressures of 25 or $28 \mathrm{~m} \mathrm{~N} \mathrm{~m}^{-1}$. For transmission electron microscopic observations, the monolayer was transferred on hydrophilic Formvar or $\mathrm{SiO}$ substrates, on which the monolayer could be transferred without a change of the aggregation state, by the horizontal lifting or upward drawing methods. Electron microscopic observations were carried out at a temperature corresponding to $T_{\mathrm{sp}}$ at which the monolayer was prepared.

\subsection{Observation of molecular arrangements in the monolayer with AFM}

Benzene solutions of lignoceric $\left(\mathrm{CH}_{3}\left(\mathrm{CH}_{2}\right)_{22} \mathrm{COOH}\right)$ and stearic $\left(\mathrm{CH}_{3}\left(\mathrm{CH}_{2}\right)_{16}\right.$ $\mathrm{COOH}$ ) acids with concentrations of $1 \times 10^{-3}$ and $3 \times 10^{-3} \mathrm{~mol} \cdot \mathrm{L}^{-1}$, respectively, were spread on pure water surface at $T_{\mathrm{sp}}$ of $293 \mathrm{~K}$. Since $T_{\mathrm{sp}}$ is below the $T_{\mathrm{m}}$ of the lignoceric acid $\left(T_{\mathrm{m}}=347 \mathrm{~K}\right)$ and the stearic acid $\left(T_{\mathrm{m}}=317 \mathrm{~K}\right)$ monolayers, those monolayers are in a crystalline state. The lignoceric acid monolayer was prepared at a surface pressure of $5 \mathrm{mN} \mathrm{m}^{-1}$ by a continuous compression at a rate of $1.7 \times 10^{-3} \mathrm{~nm}^{2}$ molecule ${ }^{-1} \mathrm{~s}^{-1}$ (Kajiyama et al 1993). The stearic acid monolayer was prepared at $23 \mathrm{mN} \mathrm{m}^{-1}$ by the continuous compression method or a multistep creep method (Kuri et al 1994). The multistep creep method is a monolayer preparation method for which the monolayer is compressed stepwise up to a higher surface pressure by alternating the compression and area creep. This procedure causes 
rearrangement of molecules in the crystalline monolayer and/or filling of the vacancies in the interfacial regions among crystalline monolayer domains, which releases the stress concentration in the monolayer. Therefore, the multistep creep method provides a mechanically stable and defect-diminished monolayer. Each monolayer was transferred onto freshly cleaved mica by a vertical dipping method. The transfer ratio for each monolayer was unity. The AFM images of the monolayers were obtained with a SFA300 (Seiko Instruments, Inc.) in air at room temperature, using a $0.8 \mu \mathrm{m}$ scanner and a silicone nitride tip on a cantilever with a small spring constant of $0.027 \mathrm{~N} \mathrm{~m}^{-1}$ (Kajiyama et al 1994, 1995). Images were recorded within $20 \mathrm{~s}$ in the "constant-height" mode; that is, feedback electronics and software were used to keep the sample height constant and measure the cantilever deflection. The applied force on imaging was evaluated to be about $10^{-10} \mathrm{~N}$ in an attractive force region, from the magnitude of cantilever deflection. This attractive force may not be the actual applied force between the tip and sample but an apparent composite force (applied force between the tip and sample, adhesion force by the water molecule at sample surface, etc.).

\section{Results and discussion}

\subsection{Novel concept for the aggregation structure of monolayers on water surface}

Infrared absorption measurements revealed that almost all the carboxylic groups of arachidic acid molecules did not dissociate on water subphase of $\mathrm{pH} 5 \cdot 8$, whereas all carboxylic groups dissociated as carboxylate ions on water subphase of $\mathrm{pH} 12 \cdot 6$.

Parts $\mathrm{a}$ and $\mathrm{b}$ of figure 1 show the $\pi-A$ isotherms for the arachidic acid monolayers on water surfaces of $\mathrm{pH} 5.8$ (pure water) and $\mathrm{pH} 12.6$ at $T_{\mathrm{sp}}$ of $303 \mathrm{~K}$, respectively, as well as the electron diffraction (ED) patterns of the monolayers at several surface pressures. In the case of a neutral state of arachidic acid (pH 5.8), the $\pi-A$ isotherm showed a sharp rise of surface pressure with decreasing surface area without any appearance of a plateau region. The ED patterns at surface pressures of 0 and $25 \mathrm{~m} \mathrm{~N} \mathrm{~m}^{-1}$ showed a crystalline arc and crystalline spot, respectively, indicating the formation of "the crystalline monolayer". Kjaer et al (1989) also reported from synchrotron X-ray diffraction studies that the arachidic acid monolayer on pure water surface revealed the crystalline phase of the monolayer at various surface pressures. The change of the ED pattern from the crystalline arc to the crystalline spot suggests that crystalline domains were fused or recrystallized at the monolayer domain interface owing to sintering behaviour caused by surface compression, resulting in the formation of larger two-dimensional crystalline domains (Tanizaki et al 1992).

In the case of a dissociated state of arachidic acid on water subphase of $\mathrm{pH} 12.6$ at $T_{\text {sp }}$ of $303 \mathrm{~K}$, a plateau region of the $\pi-A$ isotherm was observed in the range $0.3-0.5 \mathrm{~nm}^{2}$ molecule ${ }^{-1}$. The ED pattern at $5 \mathrm{mN} \mathrm{m}^{-1}$ showed an amorphous halo, whereas those at 12 and $28 \mathrm{~m} \mathrm{~N} \mathrm{~m}^{-1}$ exhibited a crystalline arc or spot. Therefore, figure $1 \mathrm{~b}$ indicates that the arachidic acid monolayer is crystallized by compression on water surface of $\mathrm{pH} \mathrm{12.6.} \mathrm{This} \mathrm{type} \mathrm{of} \mathrm{monolayer} \mathrm{has} \mathrm{been} \mathrm{classified} \mathrm{as} \mathrm{"the} \mathrm{compress-}$ ing crystallized monolayer". It is clearly concluded from parts a and b of figure 1 that amphiphile molecules form the crystalline monolayer and the compressing crystallized 

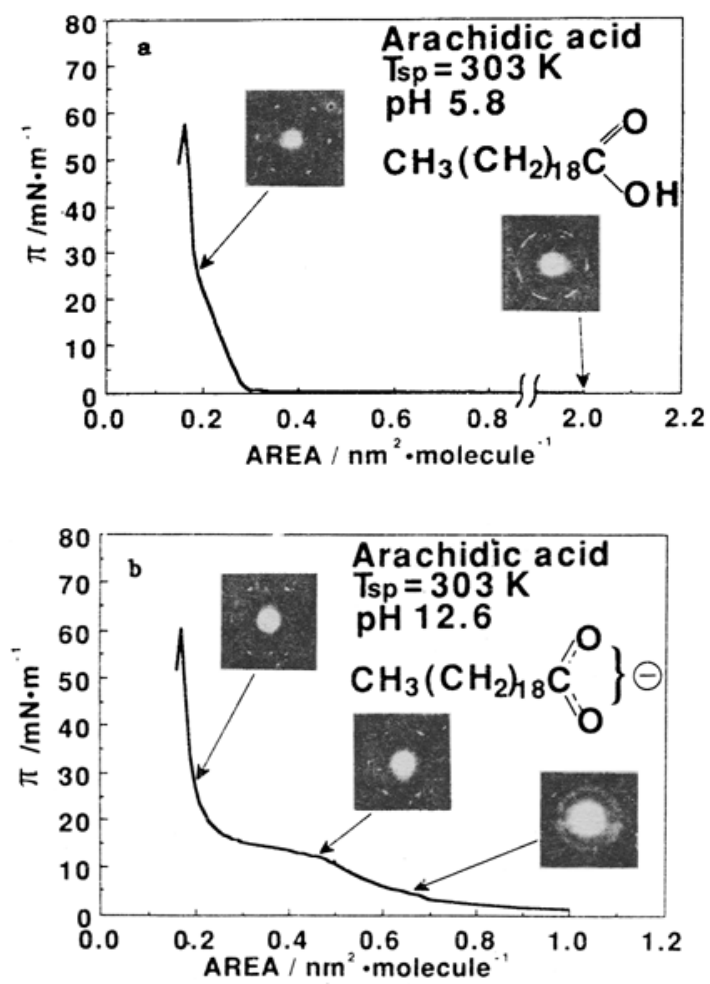

Figure 1. $\pi-A$ isotherm and ED patterns of arachidic acid monolayers at a $T_{\mathrm{sp}}$ of $303 \mathrm{~K}$ on water subphase of pH 5.8 (a) and pH $12 \cdot 6$ (b).

monolayer at $T_{\mathrm{sp}}$ below $T_{\mathrm{m}}$ in the case of a neutral state (may be the low degree of ionic dissociation) and a highly dissociated state of polar groups, respectively.

Figure 2 shows the classification based on the aggregation structure of monolayers with respect to thermal $\left(T_{\mathrm{sp}}, T_{\alpha_{\mathrm{c}}}, T_{\mathrm{m}}\right)$ and chemical (the degree of ionic dissociation of hydrophilic group) factors. This figure is divided into four quadrants by two axes of $T_{s p}$ and the repulsive force among hydrophilic groups. In the case of amphiphiles with a nonionic hydrophilic group (corresponding to the third and fourth quadrants), isolated domains grown right after spreading a solution on the water surface are gathered to be a morphologically homogeneous monolayer by compression. Then, at $T_{\mathrm{sp}}$ below $T_{\mathrm{m}}$ (the third quadrant), the monolayer is in a crystalline phase which is designated "the crystalline monolayer". The crystalline monolayer is further classified into two types: crystalline domains are assembled as a large homogeneous crystalline monolayer due to a surface compression-induced sintering at the interfacial region among monolayer domains at $T_{s p}$ below the crystalline relaxation temperature $\left(T_{\alpha_{\mathrm{c}}}\right)$, and also crystalline domains are gathered without any special orientation among domains above $T_{\alpha_{c}}$. The crystalline relaxation phenomena correspond to a change from elastic to viscoelastic characteristics in a crystalline phase due to a remarkable increase from anharmonic thermal vibration (Takayanagi and Matsuo 1967; Kajiyama et al 1974). At $T_{s p}$ above $T_{m}$ (the fourth quadrant), the monolayer is in an amorphous (noncrystalline) phase which is designated "the amorphous monolayer". In the case of amphiphiles with an ionic hydrophilic group (the first and second 
quadrants), a distinct domain structure is not formed at lower surface pressure owing to an electrostatic repulsion among polar head groups. At $T_{\mathrm{sp}}$ below $T_{\mathrm{m}}$ (the second quadrant), amphiphile molecules form a large homogeneous crystallized monolayer $\left(T_{\mathrm{sp}}<T_{\alpha_{\mathrm{c}}}\right)$ or an assembly of crystallized domains $\left(T_{\alpha_{\mathrm{c}}}<T_{\mathrm{sp}}<T_{\mathrm{m}}\right)$ owing to the contribution of the van der Waals force with an increase of surface pressure. On the other hand, at $T_{\mathrm{sp}}$ above $T_{\mathrm{m}}$ (the first quadrant), the monolayer is not crystallized by compression owing to fairly active thermal molecular motion.

\subsection{Direct observation of molecular arrangements in fatty acid monolayers with AFM}

Figure 3a shows a nonfiltered AFM image of the lignoceric acid crystalline monolayer,

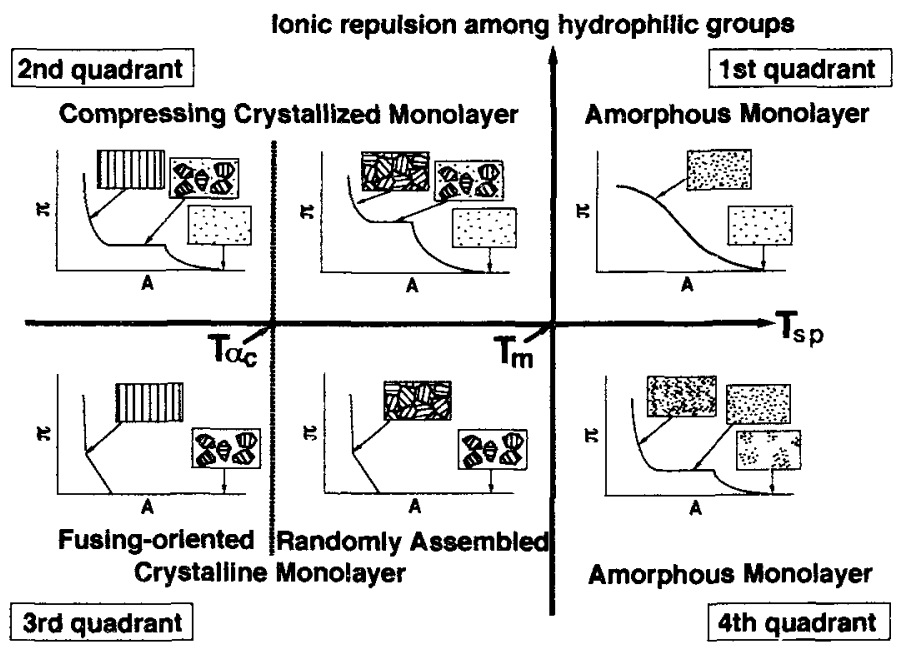

Figure 2. Classification of the aggregation stipucture of a monolayer on water surface.
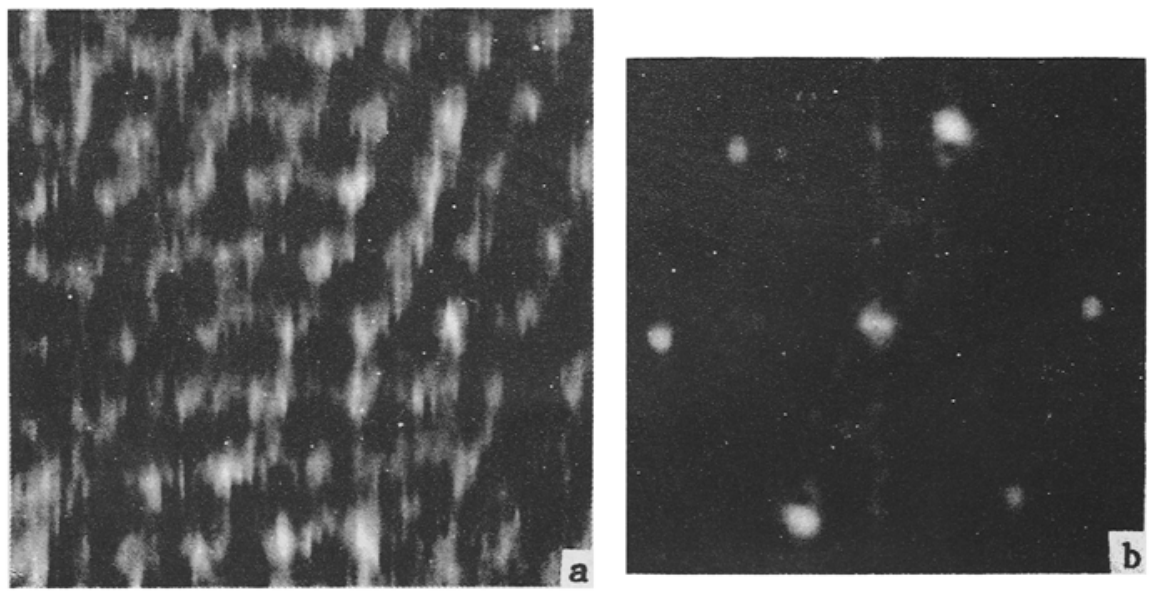

Figure 3. (a) Nonfiltered AFM image of a lignoceric acid monolayer on a scan area of $4 \times 4 \mathrm{~nm}^{2}$. (b) $2 \mathrm{D}$-FFT spectrum of (a) 
which was prepared at $5 \mathrm{mN} \mathrm{m}^{-1}$ by the continuous compression method, on a scan area of $4 \times 4 \mathrm{~nm}^{2}$. The AFM image is given in a top-view presentation in which the brighter and darker portions correspond to higher and lower regions of the monolayer surface, respectively. Though scanning was done repeatedly, the monolayer was not damaged by the tip. However, a hole could be artificially pierced through the monolayer with an applied force stronger than $10^{-9} \mathrm{~N}$. The hole was about $3 \mathrm{~nm}$ deep, being comparable with the calculated molecular length based on the CPK model, in other words, the thickness of the lignoceric acid monolayer. It is reasonable to expect that the brighter portion in the AFM image represents the single methyl group of the lignoceric acid molecule, because the hydrophobic part of the lignoceric acid molecule was oriented toward air by the vertical dipping method. The AFM image exhibits that lignoceric acid molecules are regularly arranged with a hexagonal array. In order to clarify the molecular arrangement in the monolayer, a twodimensional fast Fourier transform (2D-FFT) treatment was carried out.

Figure $3 \mathrm{~b}$ shows the 2D-FFT spectrum of the image shown in figure $3 \mathrm{a}$. The bright spots in the 2D-FFT spectrum exhibits a hexagonal pattern with the (10) spacing of $0.43 \mathrm{~nm}$. (10) represents the two-dimensional lattice plane with Miller indices of $h=1$ and $k=0$. The magnitude of (10) spacing which was evaluated from the 2D-FFT spectrum agrees well with the spacing of $0.43 \mathrm{~nm}$ which was estimated from the ED pattern of the lignoceric acid monolayer, and also this magnitude is quite different from the $0.46 \mathrm{~nm}$ spacing of a mica substrate calculated from the AFM image (figure 4). Moreover, the molecular occupied area of the lignoceric acid molecule in the monolayer which was evaluated from the AFM image and the ED pattern was $0.21 \mathrm{~nm}^{2}$ molecule ${ }^{-1}$. This magnitude is close to $0.25 \mathrm{~nm}^{2}$ molecule ${ }^{-1}$ obtained on the basis of $\pi-A$ isotherm measurements. Therefore, it is reasonable to conclude from figures 3 and 4 that the brighter portion in the AFM image of figure 3 a represents the

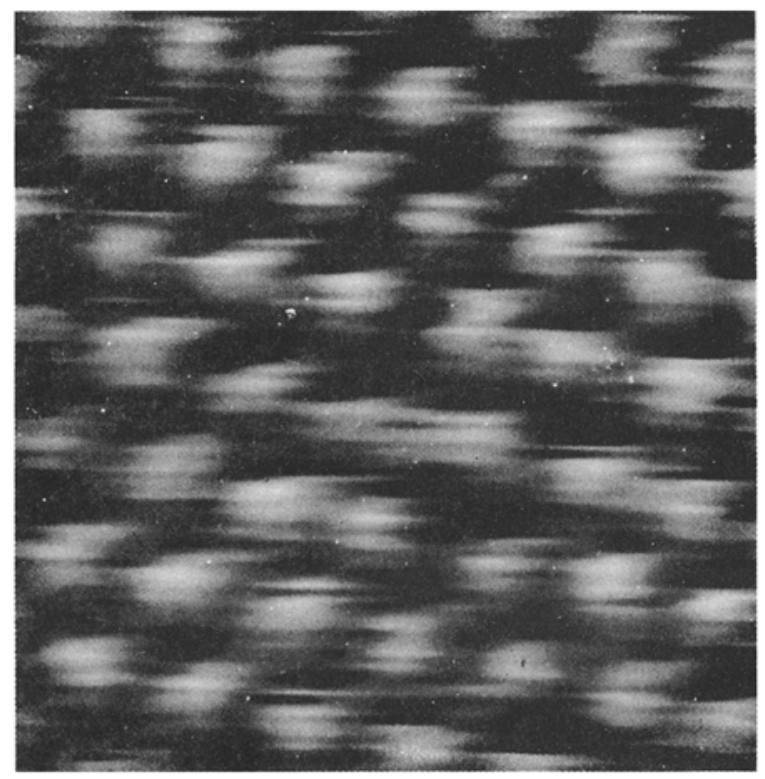

Figure 4. Nonfiltered AFM image of a mica substrate on a scan area of $4 \times 4 \mathrm{~nm}^{2}$. 


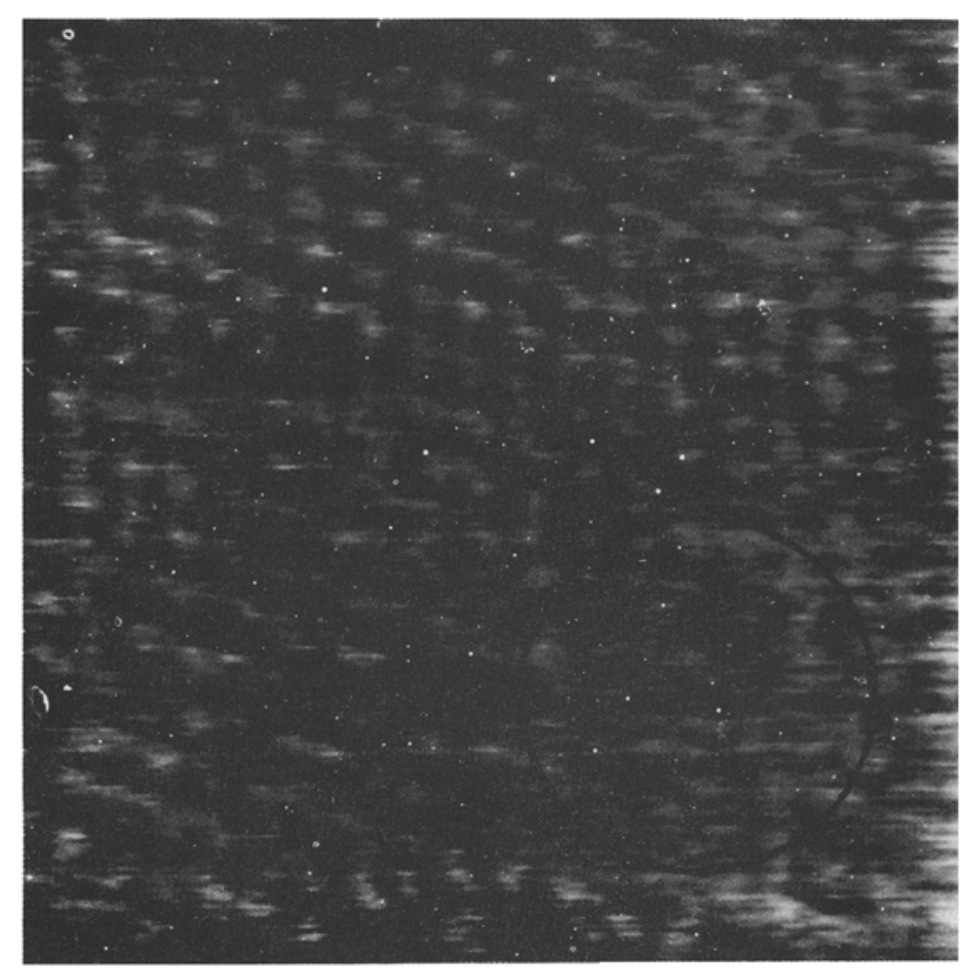

Figure 5. Nontiltered AFM image of a lignoceric acid monolayer on a scan area or $9 \times 9 \mathrm{~nm}$.

single methyl group of the lignoceric acid molecule in the monolayer and also that lignoceric acid moiecules are regularly arranged in a hexagonal array.

Figure 5 shows a nonfiltered AFM image of the lignoceric acid monolayer on a larger area scan of $9 \times 9 \mathrm{~nm}^{2}$. A regularly periodic hexagonal array in the AFM image was extende 1 over :bout $10 \mathrm{~nm}$. The range of the periodic hexagona! array was comparable to the nagnitude of crystallographical continuity which was evaluated by a single line method (Hofnann and Walenta 1987: Oishi et al 1994) based on Fourier analysis of the ED profile of the lignoceric acid monolayer. As shown by the circle in figure 5, the hexagonal array of lignoceric acid molecules was locally disordered. Thus, the molecular-resolution AFM image of the lignoceric acid monolayer was nondestructively obtaines and exhibited a two-dimensional periodic structure with locally disordered molecular arrangements.

The nondestructive AFM observation of the lignoceric acid monolayer was successful, as shown in figures 3 and 5 . However. it was impossible to obtain a molecular-resolution AFM image of the ignoceric acid monolayer which was continuously compressed up to surface pressures higher than $10 \mathrm{mN} \mathrm{m}^{-1}$ because of monolayer destruction during the AFM scan. In the case of the crystalline monolayer, the crystalline domains grown right after spreading a solution are assembled into a morphologically homogeneous monolayer during comp ession. However, the vacancies at a molecular level may remain at the interfaces am ong the crystalline domains in the monolayer when the monolayer is prepared by the continuous compression method. These vacancies cause stress 

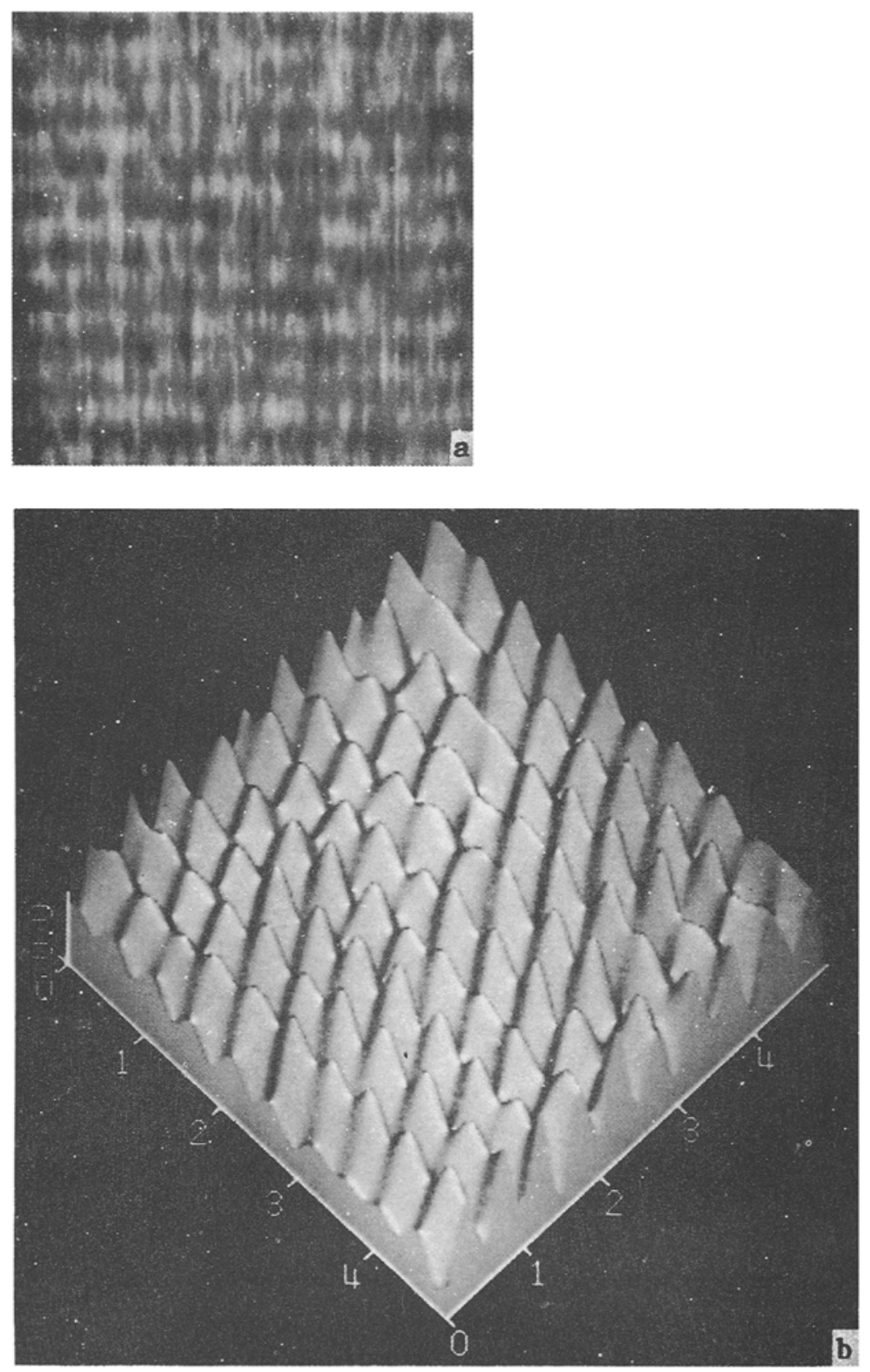

Figure 6. (a) Nontiltered AFM image of a stearic acid monolayer on a scan area 0 $5 \times 5 \mathrm{~nm}^{2}$ (b) A flitered AFM image of (a).

concentration in the monolayer under a continuous compression. Since large stress locally concentrates in the monolayer at higher surface pressures, such a monolayer is mechanically unstable. This mechanical instability may be the reason why the monolayer continuously compressed up to a high surface pressure, for example $10 \mathrm{mN} \mathrm{m}^{-1}$, 
was easily collapsed by the applied force on the AFM scan. This argument was justified in the nondestructive AFM image of the monolayer prepared at a fairly higher surface pressure, for example $23 \mathrm{mN} \mathrm{m}^{-1}$, by the multistep creep method, because stress concentration in the monolayer was almost completely released.

Figure 6a shows a nonfiltered AFM image of the stearic acid crystalline monolayer prepared by the multistep creep method with a scan area of $5 \times 5 \mathrm{~nm}^{2}$. Though scanning was done repeatedly on the stearic acid monolayer prepared at the high surface pressure of $23 \mathrm{mN} \mathrm{m}^{-1}$ by the multistep creep method, the monolayer was not damaged by the tip. On the other hand, the monolayer prepared at the same surface pressure by the continuous compression method was easily collapsed by the tip on scanning. This collapse may be caused by stress concentration at vacancies among crystalline domains in the monolayer. In order to reduce the noise component in figure 6a, a digital filtering treatment was used to get the Fourier-transformed image. Figure $6 \mathrm{~b}$ shows a filtered AFM image of the monolayer. A higher region in the AFM image represents the single methyl group of the stearic acid molecules, which are regularly arranged in a hexagonal array with a (10) spacing of $0.42 \mathrm{~nm}$. This magnitude agrees with the spacing of $0.42 \mathrm{~nm}$ estimated from the ED pattern of the stearic acid monolayer. When the area scan was enlarged, the regularly periodic hexagonal array was extended over $20 \times 20 \mathrm{~nm}^{2}$ as shown in figure 7 . This magnitude is comparable to the magnitude of crystallographical continuity evaluated on the basis of the single line method. Further, no distinct molecular disordered region was observed in

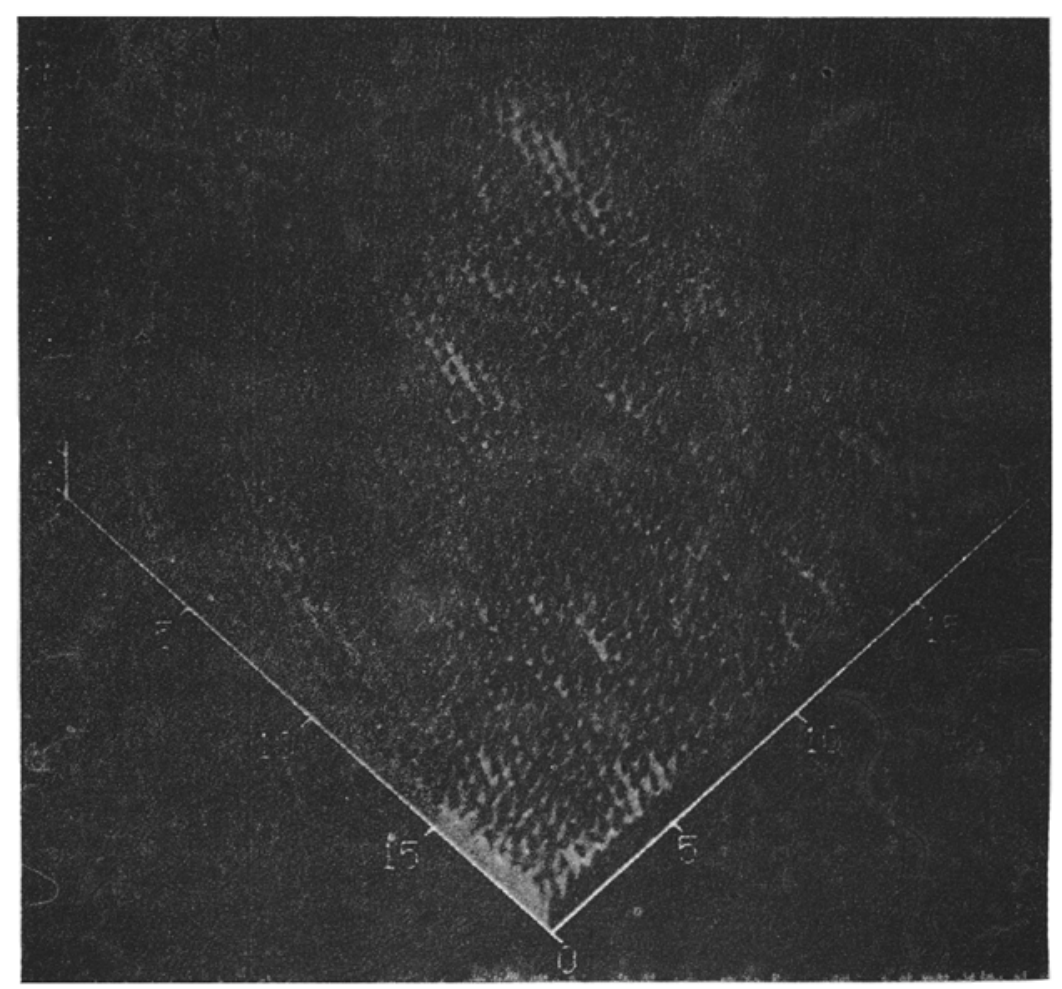

Figure 7, A tiltered AFM inage of a sterric acid monoliver on at car arta of $20 \times 20 \mathrm{~nm}^{2}$. 
the scan area of $20 \times 20 \mathrm{~nm}^{2}$ in the AFM image. The larger crystallographical continuity and extended regular molecular arrangement of the stearic acid monolayer are ascribed to effective sintering at interfaces among crystalline domains by the multistep creep method.

\section{Conclusion}

The aggregation structure of fatty acid monolayers on water subphases of different pH's was investigated by TEM observations. The aggregation structure of monolayers on water surface was systematically classified into "the crystalline monolayer", "the amorphous monolayer" and "the compressing crystallized monolayer" with respect to thermal and chemical factors. The molecular-resolution AFM image of the fatty acid monolayer was successfully taken using a mechanically stable monolayer. The fatty acid monolayer with a remarkable mechanical stability for nondestructive AFM observations was prepared at a higher surface pressure by the multistep creep method and at a lower surface pressure by the continuous compression method.

\section{References}

Gaines Jr G L 1966 Insoluble monolayers at liquid-gas interface (New York: Interscience) ch. 4

Hofmann D and Walenta E 1987 Polymer 281298

Kajiyama T and Takayanagi M 1974 J. Macromol. Sci. Phys. B10 131

Kajiyama T, Okada T and Takayanagi M 1974 J. Macromol. Sci. Phys. B9 35

Kajiyama T, Oishi Y, Uchida M, Morotomi N, Ishikawa J and Tanimoto Y 1992a Bull. Chem. Soc. Jpn. 65864

Kajiyama T, Oishi Y, Uchida M, Tanimoto Y and Kozuru H 1992b Langmuir 81563

Kajiyama T, Oishi Y, Hirose F, Shuto $K$ and Kuri T 1993a Chem. Lett. 1121

Kajiyama T, Oishi Y, Uchida M and Takashima Y 1993b Langmuir 91978

Kajiyama T, Zhang L, Uchida M, Oishi Y and Takahara A 1993c Langmuir 9760

Kajiyama T, Oishi Y, Hirose F, Shuto K and Kuri T 1994 Langmuir 101297

Kajiyama T, Oishi Y, Suehiro K. Hirose F and Kuri T 1995 Chem. Lett. 241

Kjaer K, Als-Nielsen J, Helm C A, Tippman-Krayer P and Möhwald H 1989 J. Phys. Chem. 933200

Kuri T, Oishi Y and Kajiyama T 1994 Trans. Mater. Res. Sac. Jpn A15 567

Oishi Y, Hirose F, Kuri T and Kajiyama T 1994 J. Vac. Sci. Technol. A12 2971

Peltonen J P K, He P and Rosenholm J B 1992 J. Am. Chem. Soc. 1147637

Schwartz D K, Viswanathan R, Garnaes J and Zasadzinski J A 1993 J. Am. Chem. Soc. 1157374

Takayanagi Mi and Matsuo M 1967 J. Macromol. Sci. Phys. B1 407

Tanizaki T, Takahara A and Kajiyama T 1991 J. Soc. Rheol. Jpn 19208 